ARTICLE

DOI: $10.1038 / \mathrm{s} 41467-017-01180-5$

\title{
MRE11 and EXO1 nucleases degrade reversed forks and elicit MUS81-dependent fork rescue in BRCA2- deficient cells
}

Delphine Lemaçon', Jessica Jackson', Annabel Quinet', Joshua R. Brickner², Shan Li ${ }^{3}$, Stephanie Yazinski ${ }^{4}$, Zhongsheng You ${ }^{3}$, Grzegorz Ira ${ }^{5}$, Lee Zou ${ }^{4}$, Nima Mosammaparast ${ }^{2}$ \& Alessandro Vindigni ${ }^{1}$

The breast cancer susceptibility proteins BRCA1 and BRCA2 have emerged as key stabilizing factors for the maintenance of replication fork integrity following replication stress. In their absence, stalled replication forks are extensively degraded by the MRE11 nuclease, leading to chemotherapeutic sensitivity. Here we report that BRCA proteins prevent nucleolytic degradation by protecting replication forks that have undergone fork reversal upon drug treatment. The unprotected regressed arms of reversed forks are the entry point for MRE11 in BRCA-deficient cells. The CtIP protein initiates MRE11-dependent degradation, which is extended by the EXO1 nuclease. Next, we show that the initial limited resection of the regressed arms establishes the substrate for MUS81 in BRCA2-deficient cells. In turn, MUS81 cleavage of regressed forks with a ssDNA tail promotes POLD3-dependent fork rescue. We propose that targeting this pathway may represent a new strategy to modulate BRCA2deficient cancer cell response to chemotherapeutics that cause fork degradation.

\footnotetext{
${ }^{1}$ Edward A. Doisy Department of Biochemistry and Molecular Biology, Saint Louis University School of Medicine, St Louis, MO 63104, USA. ${ }^{2}$ Department of Pathology and Immunology, Division of Laboratory and Genomic Medicine, Washington University School of Medicine, St Louis, MO 63110, USA.

${ }^{3}$ Department of Cell Biology and Physiology, Washington University School of Medicine, Campus Box 8228, 660S. Euclid Ave., St Louis, MO 63110, USA.

${ }^{4}$ Massachusetts General Hospital Cancer Center, Harvard Medical School, Boston, MA 02129, USA. ${ }^{5}$ Department of Molecular and Human Genetics, Baylor College of Medicine, One Baylor Plaza, Houston, TX 77030, USA. Correspondence and requests for materials should be addressed to A.V. (email: avindign@slu.edu)
} 
G ermline mutations in the Breast Cancer Susceptibility genes BRCA1/BRCA2 account for the vast majority of familial breast cancer cases ${ }^{1-4}$. Aside from their wellestablished roles in homologous recombination (HR), BRCA proteins are emerging as key factors required for the maintenance of replication fork stability following replication stress induction $^{5-8}$. In their absence, replication forks are extensively degraded by the MRE11 nuclease. MRE11-dependent degradation of replication forks observed in the absence of BRCA proteins leads to long stretches of ssDNA $(>4-5 \mathrm{~kb})$ and is emerging as one of the leading causes of the sensitivity to therapies that target DNA or that inhibit specific repair pathways such as PARP inhibitors ${ }^{5}$. The mechanism leading to this extensive fork degradation phenotype in the absence of BRCA1 or BRCA2 remains unclear. For example, the exact structure(s) of the replication intermediates targeted by nucleases in BRCA-deficient cells is unknown. Moreover, MRE11 has limited nucleolytic activity $^{9}$ and is unlikely to be the only nuclease responsible for degrading several $\mathrm{kb}$ of DNA in BRCA-deficient cells. Finally, the fate of the extensively resected forks upon drug removal has never been investigated in detail, even though it is tightly linked to the increased chromosomal aberrations and DNA damage sensitivity of BRCA-deficient cells.

Replication fork reversal is a key protective mechanism that allows replication forks to reverse their course when they encounter DNA lesions ${ }^{10-14}$. Interestingly, the same HR factors controlling MRE11 nuclease activity and ssDNA accumulation are also emerging as crucial players involved in fork remodeling ${ }^{14-16}$. In particular, the central recombinase RAD51 is essential for fork reversal upon chemotherapeutic treatment ${ }^{14}$. By analogy with its bacterial homologue RecA, RAD51 may be recruited to ssDNA stretches formed at replication fork junctions and promote the initial step of fork reversal by invading the complementary strand. In this context, HR proteins may also be required to stabilize forks in their reversed state by protecting the double-stranded end of the regressed arm from nucleolytic degradation.

In this study, we combine electron microscopy (EM) with genome-wide single-molecule DNA fiber approaches to define the mechanism by which the BRCA proteins protect replication forks from nucleolytic degradation following replication stress induction. We show that the main function of BRCA proteins in this context is to protect the regressed arms of replication forks that have reversed upon drug treatment from nucleolytic degradation. In their absence, CtIP initiates the MRE11-dependent degradation of the unprotected regressed arms and EXO1 contributes to extend fork degradation. Next, we investigate how cells cope with these extensively resected forks upon drug removal. In particular, we find that MUS81 cleavage rescues the resected forks in BRCA2-, but not BRCA1-deficient cells through a break-induced replication (BIR)-like mechanism mediated by POLD3dependent DNA synthesis. Our findings revisit the functions of central HR factors in DNA replication and are crucial to understanding how targeting BIR-dependent pathways can modulate current chemotherapeutic modalities.

EXO1 contributes to fork resection in BRCA-deficient cells. Two distinct pathways act redundantly to mediate processive double-strand break (DSB) resection downstream from the MRE11-RAD50-NBS1 (MRN) and CtIP factors in eukaryotic cells: one requires DNA2 and the other EXO1 ${ }^{17-21}$. We sought to investigate whether DNA2 and EXO1 also contribute to the extended fork degradation phenotype of BRCA1- or BRCA2deficient cells following genotoxic stress induction. We knocked down EXO1 or DNA2 in different cell lines, including the
BRCA2-mutant ovarian cancer cells PEO1 (and the isogenic PEO4 cells, which contain a second point mutation restoring BRCA2 function), the Fanconi anemia BRCA2-mutant line EUFA423 (and its derivative expressing wild-type BRCA2), the BRCA1 mutant ovarian cancer cell line UWB1.289 (and its complemented derivative expressing wild-type BRCA1), plus the human osteoscarcoma U2OS cells, which were siRNA-depleted for BRCA1 or BRCA2. Nucleolytic resection following replication fork stalling was monitored by pulse-labeling cells with the first thymidine analog IdU (red label) followed by treatment with hydroxyurea (HU) and concomitant labeling with the second thymidine analog, CldU (green label) (Fig. 1a). Shortening of the first tract was measured as a readout of degradation only on forks characterized by contiguous IdU-CldU signals (and not on forks that have only the IdU label) to ensure that the shortening phenotype is indeed due to nucleolytic resection of stalled replication forks and not to premature termination events ${ }^{22}$. Upon $\mathrm{HU}$ treatment, BRCA1- and BRCA2-deficient cells showed a marked reduction (30-50\% corresponding to $>5 \mathrm{~kb}$ of DNA) in the IdU tract length (Fig. 1b, c and Supplementary Fig. 1a). Moreover, MRE11 inhibition or knockdown partially rescued fork degradation consistent with the previous data ${ }^{5-7}$ (Fig. 1b, c and Supplementary Fig. 1). Analogous results were obtained using an alternative labeling scheme, where $\mathrm{HU}$ was added after thymidine labeling, suggesting that the results were not affected by the particular labeling scheme used in our work (Supplementary Fig. 1b). EXO1 knockdown by two different siRNAs yielded the same fork protection phenotype observed with MRE11 inhibition, indicating that EXO1 also contributes to fork degradation in BRCA1- and BRCA2-deficient cells upon HU treatment (Fig. 1 and Supplementary Figs. 1a-e and 2a, b). Interestingly, combined ablation of MRE11 and EXO1 activities further rescued the fork degradation phenotype of BRCA-deficient cells, suggesting that the two nucleases may be able to act independently on the stalled replication intermediates. The same results were obtained by treating BRCA2-deficient cells with DNA damaging agents such as cisplatin or UV-C, supporting the notion that different genotoxic agents trigger a similar fork resection mechanism whereby MRE11 and EXO1 extensively degrade replication forks in the absence of key HR factors (Supplementary Fig. 3a, b). Conversely, DNA2 knockdown did not restore fork protection (Supplementary Fig. $2 \mathrm{c}-\mathrm{g}$ ), in agreement with previous findings ${ }^{23}$. The same results were recapitulated by treating BRCA2-deficient cells with the NSC-105808 DNA2 inhibitor ${ }^{24}$ (Supplementary Fig. 2e). Taken together, these results suggest that human EXO1, but not DNA2, contributes to replication fork degradation in BRCA1and BRCA2-deficient cells.

MRE11 and EXO1 target reversed forks in BRCA-deficient cells. Next, we sought to investigate the actual structure(s) of the replication intermediates targeted by MRE11 and EXO1 in BRCA1- or BRCA2-deficient cells. We visualized the fine architecture of the replication intermediates using a combination of in vivo psoralen cross-linking and $\mathrm{EM}^{25}$ (Fig. 2a). Our analysis showed a substantial fraction of reversed replication forks $(\sim 25 \%$ of molecules analyzed) in control U2OS cells treated with $4 \mathrm{mM}$ HU, consistent with previous data ${ }^{14,26}$ (Fig. 2b). BRCA1- or BRCA2-knockdown resulted in a significantly lower frequency of fork reversal events $(\sim 10$ and $11 \%$, respectively) compared to HU-treated control cells, suggesting that BRCA proteins are required either to promote fork reversal or to prevent nucleolytic processing of forks that have already reversed following $\mathrm{HU}$ treatment. To distinguish between these two possibilities, we repeated the EM analysis while inhibiting MRE11 activity or knocking down EXO1 in BRCA-deficient cells. We found that 
ablation of either nuclease rescues the frequency of reversed forks to levels observed in control cells, suggesting that BRCA proteins protect forks that have already undergone fork reversal from nucleolytic degradation. Similar results were obtained by inhibiting MRE11 in BRCA2-mutant PEO1 cells (Fig. 2c). Timecourse experiments performed by treating BRCA2-deficient $\mathrm{U} 2 \mathrm{OS}$ cells with $\mathrm{HU}$ for 0,30 , or $120 \mathrm{~min}$ indicated that fork reversal precedes fork degradation, as predicted by our model (Fig. 2d-f). Collectively, these results suggest that BRCA proteins protect reversed forks from nucleolytic degradation and that the unprotected reversed forks are the entry point for MRE11 and EXO1 in BRCA1- and BRCA2-deficient cells.
CtIP initiates MRE11-dependent resection of reversed forks. The regressed arm of a reversed replication fork resembles by all means a one-ended DSB. Previous biochemical studies showed that the $5^{\prime}-3^{\prime}$ endonuclease activity of MRE11 initiates the resection process and that CtIP is required to promote MRE11 endonuclease activity at the $5^{\prime}$ strand ${ }^{27}$. Resection is then continued by the $5^{\prime}-3^{\prime}$ exonuclease activity of EXO1 ${ }^{19}, 28,29$. As an alternative approach to confirm that MRE11-dependent resection starts from the regressed arm of reversed replication forks, we repeated the DNA fiber experiments with CtIP knockdown cells and found that CtIP loss prevents fork degradation in BRCA2deficient cells (Supplementary Fig. 3c, d). These data suggest that the same pathway that initiates DSB resection in the context of
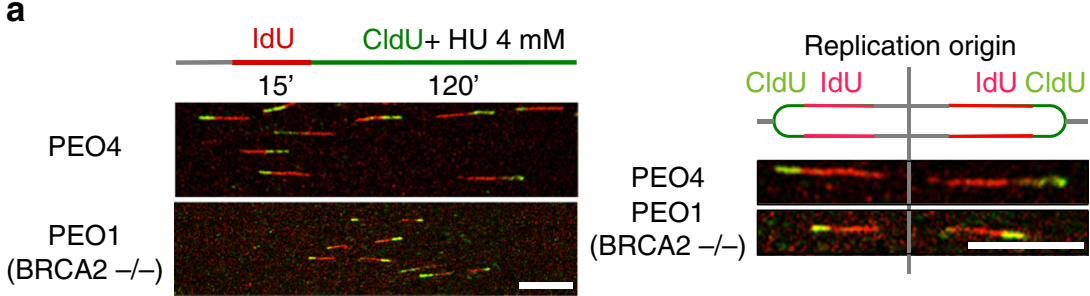

b

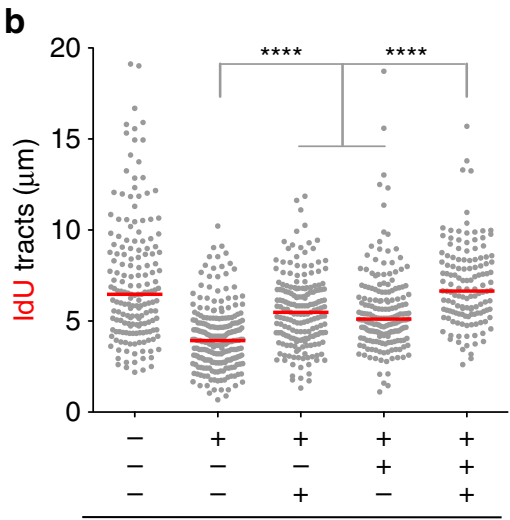

U2OS SiBRCA2

c

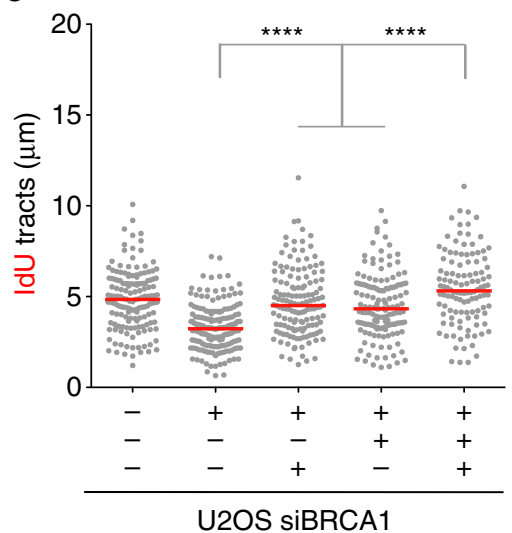

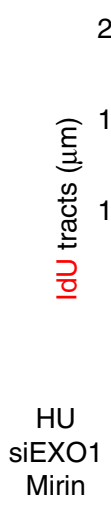

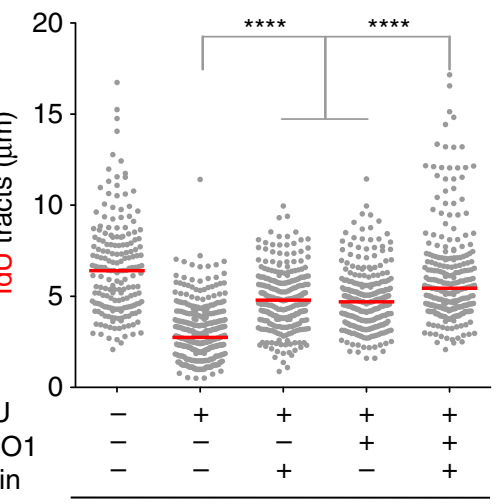

PEO1 (BRCA2-/-)

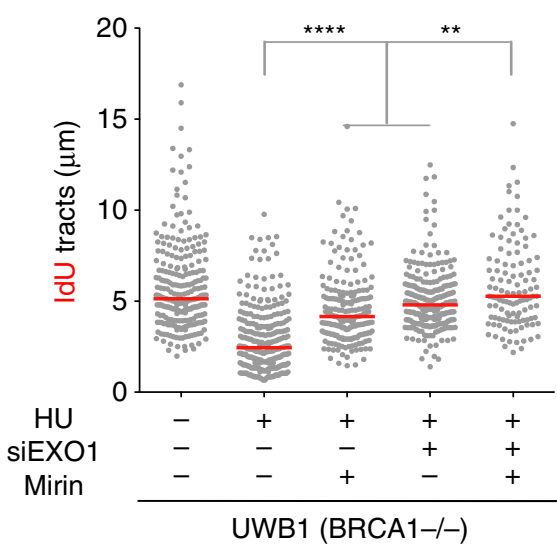

Fig. 1 MRE11 and EXO1 mediate extended nascent strand degradation in HU-treated BRCA1- and BRCA2-deficient cancer cells. a Schematic of the singlemolecule DNA fiber tract analysis and representative DNA fiber images of PEO4 and PEO1 cells treated with HU ( 4 mM) for 120 min. IdU, red; CldU, green. Scale bar, $50 \mu \mathrm{m}$. b Size distribution of IdU tract length in BRCA2-deficient U2OS (left) and PEO1 (right) cells in the presence and absence of HU. Cells were transfected with control siRNA or EXO1 siRNA before IdU and CldU labeling. Mirin $(50 \mu \mathrm{M})$ was added concomitantly with HU treatment, as indicated. Out of 3 repeats; $n \geq 250$ tracts scored for each data set. Bars represent the median. Statistics: Mann-Whitney; ${ }^{\star \star \star \star} P<0.0001$. c Size distribution of IdU tract length in BRCA1-deficient U2OS (left) and UWB1 (right) cells in the presence and absence of HU. Cells were transfected with control siRNA or EXO1 siRNA before IdU and CldU labeling. Mirin $(50 \mu \mathrm{M})$ was added concomitantly with $\mathrm{HU}$ treatment, as indicated. Out of 2 repeats; $n \geq$ 250 tracts scored for each data set. Bars represent the median. Statistics: Mann-Whitney; ${ }^{\star \star} P<0.01 ;{ }^{\star \star \star \star} P<0.0001$ 
a

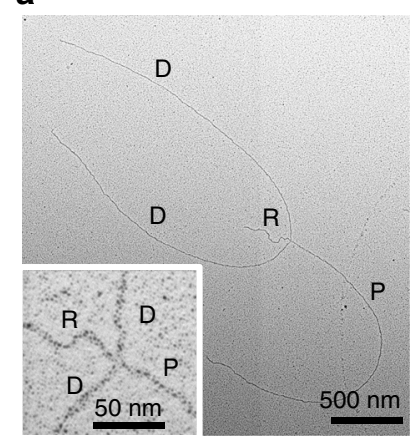

C

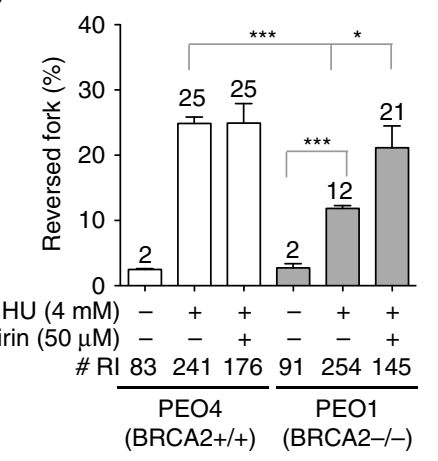

b

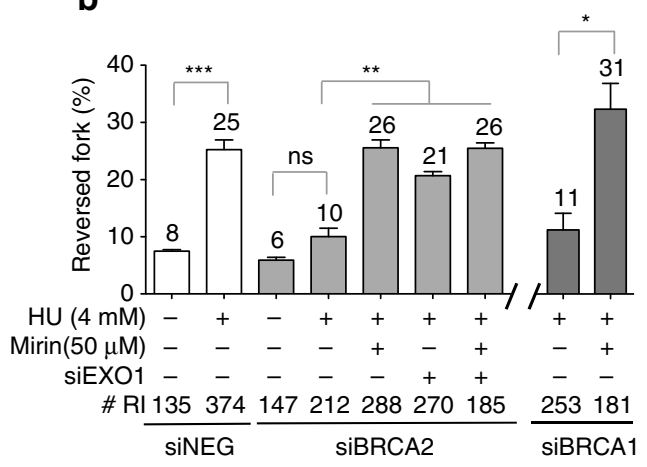

d

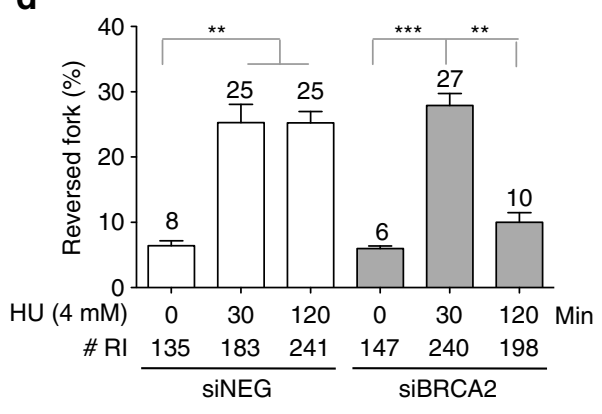

e

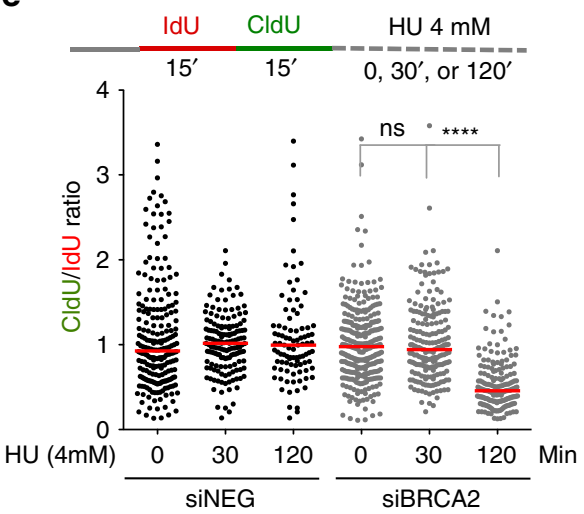

f

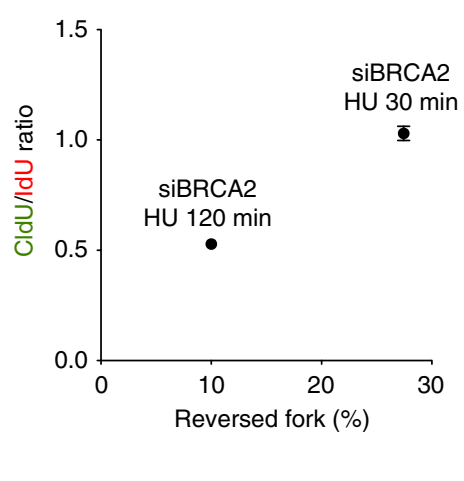

Fig. 2 BRCA1 and BRCA2 protect reversed replication forks from MRE11- and EXO1-dependent degradation following HU treatment. a Representative electron micrograph of a reversed fork observed on genomic DNA upon $\mathrm{HU}$ treatment. Inset, magnified four-way junction at the reversed replication fork. D daugher strand, P parental strand, R reversed arm. b Frequency of fork reversal in BRCA1- and BRCA2-deficient U2OS cells treated with $4 \mathrm{mM} \mathrm{HU}$ for $5 \mathrm{~h}$. Cells were transfected with control siRNA (siNEG) or EXO1 siRNA. Mirin $(50 \mu \mathrm{M})$ was added concomitantly with HU, as indicated. The percentage values are indicated on the top of the bar. "\# RI" indicates the number of analyzed replication intermediates. Mean shown, $n=3$. Errors, S.E.M. Statistics: unpaired $t$ test; ${ }^{\star} P<0.05$; ${ }^{\star \star} P<0.01 ;{ }^{\star \star \star} P<0.001$. c Frequency of fork reversal in BRCA2-proficient PEO4 and BRCA2-deficient PEO1 cells treated with $4 \mathrm{mM}$ HU for $5 \mathrm{~h}$. Mirin $(50 \mu \mathrm{M})$ was added concomitantly with $\mathrm{HU}$, as indicated. The percentage values are indicated on the top of the bar. "\# RI" indicates the number of analyzed replication intermediates. Mean shown, $n=3$. Errors, S.E.M. Statistics: unpaired $t$ test; ${ }^{\star} p<0.05$; ${ }^{\star \star \star} p<0.001$. d Frequency of fork reversal in BRCA2-deficient U2OS cells treated with $4 \mathrm{mM} \mathrm{HU}$ for 0,30 , or 120 min. The percentage values are indicated on the top of the bar. "\# RI" indicates the number of analyzed replication intermediates. Mean shown, $n=3$. Errors, S.E.M. Statistics: unpaired $t$ test; ${ }^{* \star} P<0.01$; ${ }^{\star \star \star} P<0.001$. e CldU/IdU tract ratio in BRCA2-deficient U2OS cells treated with $4 \mathrm{mM} \mathrm{HU}$ for 0,30 , or 120 min. Out of 3 repeats; $n \geq 250$ tracts scored for each data set. Bars represent the median. Statistics: Mann-Whitney; ${ }^{\star \star \star \star} P<0.0001$. $\mathbf{f}$ Plot the percentages of reversed forks as a function of mean values of the CldU/IdU ratios measured after treating BRCA2-deficient U2OS cells with $4 \mathrm{mM} \mathrm{HU}$ for 30 or 120 min. Errors, S.E.M.

$\mathrm{HR}$ is required to process the open dsDNA end of the regressed arm. To further validate this conclusion, we reasoned that degradation should not take place in a genetic background that prevents reversed fork formation-i.e., RAD51 knockdown ${ }^{14}$. Indeed, loss of RAD51 suppressed fork degradation in BRCA2depleted U2OS cells, confirming that RAD51 acts upstream of BRCA2 to promote reversed fork formation (Fig. 3a, b). In addition, RAD51 depletion severely compromised fork restart, suggesting that fork remodeling is an essential requirement for efficient resumption of DNA synthesis upon HU removal (Figs. 3c, d).

Resection does not affect fork restart upon BRCA2 loss. The extended nucleolytic degradation of stalled replication 
intermediates in BRCA1- and BRCA2-deficient cells leads to increased chromosomal aberrations and genome instability ${ }^{5,6}$. However, the molecular mechanism linking fork degradation with chromosomal instability remains elusive. Thereby, we set out to investigate how cells cope with these extensively degraded forks focusing on BRCA2-deficient cells. Fork degradation associated with BRCA2 loss did not significantly affect fork restart (Fig. 3e), in agreement with previous findings 5,6 . However, by increasing the timing of CldU labeling from 15 to $90 \mathrm{~min}$, we found that thymidine incorporation after $\mathrm{HU}$ removal was reduced in BRCA2-deficient compared to BRCA2-proficient cells (Fig. 3f). This reduction in the CldU tract length suggests that BRCA2 loss, though not affecting the number of restarting forks, either impairs fork progression after restart or causes a delayed restart of the resected forks. Ablation of MRE11 and/or EXO1 activity rescued CldU tract length, suggesting that the extended nascent strand degradation associated with BRCA2 loss is the leading cause of the observed defect in fork progression or timing of

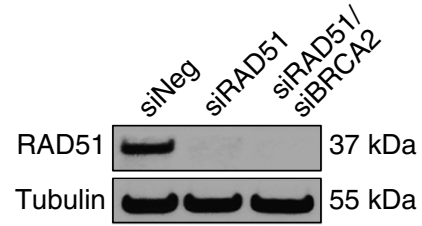

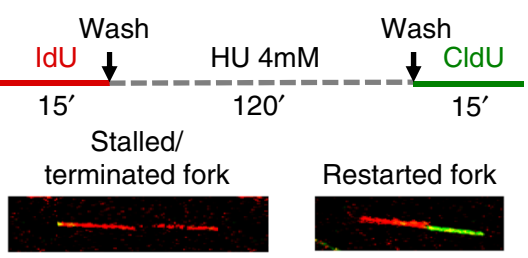

d b

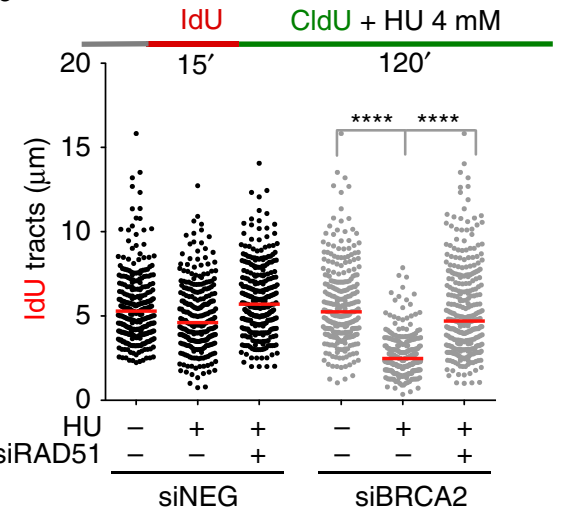

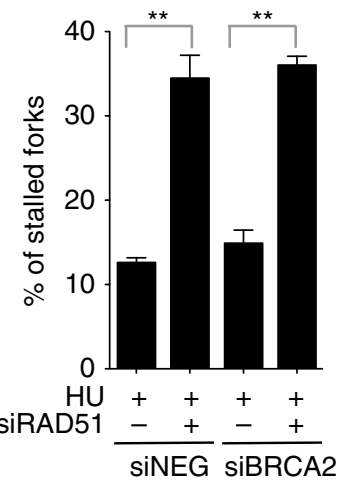

Wash

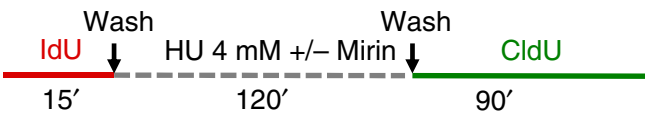

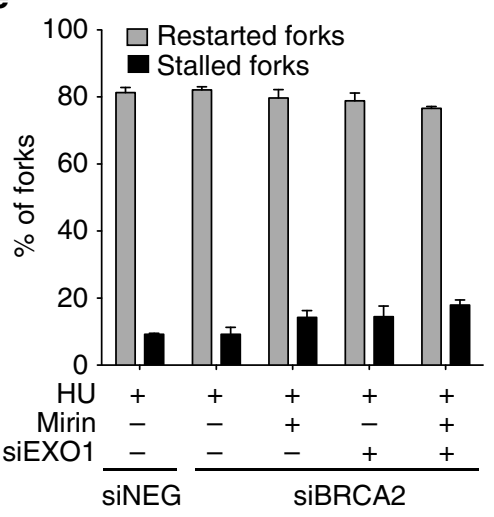

f

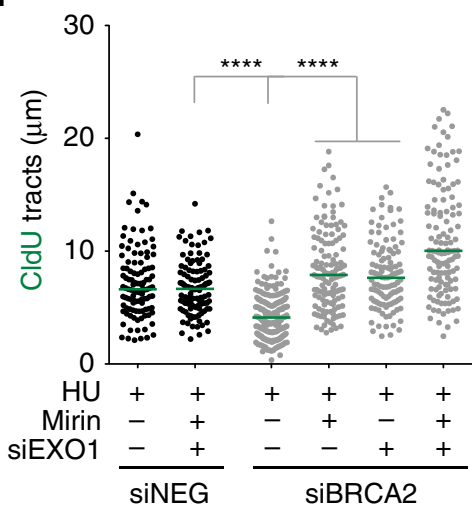

9

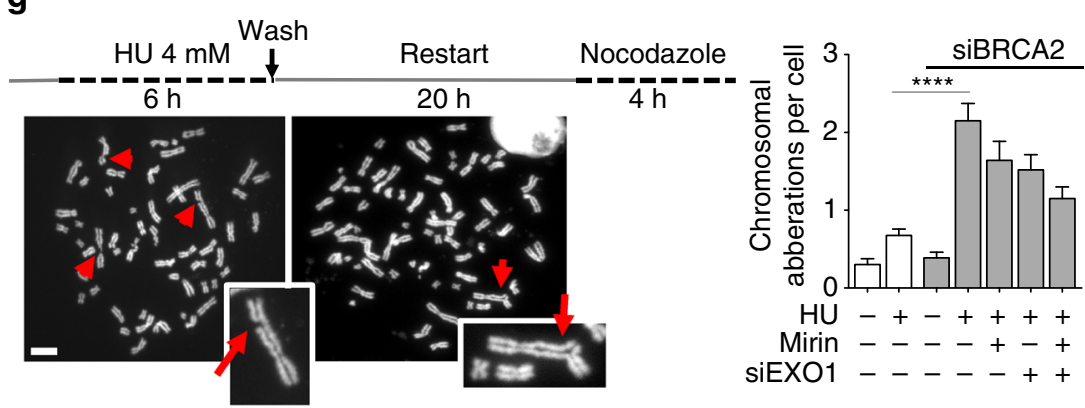


restart (Fig. 3f). Moreover, BRCA2 loss led to increased chromosomal aberrations when cells were challenged with $\mathrm{HU}$, and this effect was again partially rescued by ablation of MRE11 and/ or EXO1 activity, supporting a link between fork degradation, defects in fork progression or restart upon drug removal and increased chromosomal aberrations (Fig. 3g).

MUS81 cleaves partially resected reversed forks. We next examined the mechanism that rescues resected forks upon drug removal. Using neutral comet assays we found that BRCA2 loss leads to DSB accumulation upon HU treatment (Figs. 4a, b). However, the frequency of DSBs decreased to control levels 15 min after HU removal, suggesting that these DSBs are quickly repaired after fork restart. Interestingly, MUS81 depletion prevented DSB accumulation in BRCA2-deficient cells (Fig. 4c). A short-interfering RNA (siRNA)-resistant MUS81 cDNA, but not a catalytically inactive version (MUS81(D338A/D339A)), restored DSB accumulation in MUS81 siRNA-depleted cells (Fig. 4c and Supplementary Fig. 3f). Therefore, we propose that MUS81 endonuclease activity is required to cleave partially resected forks, leading to transient DSB accumulation in the absence of BRCA2. Our observation that MUS81 loss did not significantly affect fork resection supports the idea that the extended fork degradation phenotype was not due to the resection of the DSBs created by MUS81 cleavage (Supplementary Fig. 3g). Moreover, the notion that MUS81 acts downstream of MRE11 and EXO1 was supported by the observation that the DSB accumulation of BRCA2deficent cells was significantly reduced in a genetic background that prevents fork resection-i.e., loss of MRE11, EXO1, or CtIP activity (Fig. 4c and Supplementary Fig. 3e).

To define the exact substrate cleaved by MUS81 in BRCA2deficient cells, we inspected the structure of the replication intermediates that accumulate in BRCA2-deficient cells upon MUS81 depletion. Our EM analysis revealed that MUS81 loss causes a dramatic increase in reversed fork frequency in BRCA2deficient cells (Fig. 5a, b). Next, we evaluated the ssDNA composition of the regressed arms by detecting local differences in filament thickness. MUS81 depletion led to a significant increase in the percentage of regressed forks that are partially or entirely single-stranded, strongly suggesting that MUS81 cleaves partially resected regressed forks with a ssDNA tail (Fig. 5b). Interestingly, MUS81 depletion also led to a significant increase in the percentage of daughter strands with ssDNA at the fork junction, suggesting that in the absence of MUS81 cleavage asymmetric fork resection continues beyond the length of the regressed arm, leading to partially resected 3-way junction structures (Fig. 5c). Of note, the small fraction of HU-induced DSBs of BRCA2-proficient cells is also rescued by the loss of
MUS81, but not of MRE11 or EXO1 (Supplementary Fig. 3h). This observation is consistent with the previous biochemical data showing that MUS81 can also cleave intact forks, although with much lower efficiency compared to flap-fork substrates ${ }^{30}$. Taken together, these results suggest that the initial resection of the regressed arms leads to the formation of reversed forks with a ssDNA flap, which are cleaved by MUS81.

MUS81 cleavage promotes POLD3-dependent DNA synthesis. To test whether MUS81-dependent cleavage of resected regressed forks is required for fork restart, we quantified the percentage of stalled forks in MUS81 and BRCA2 co-depleted cells (Fig. 5d). MUS81 loss slightly increased fork stalling in BRCA2-proficient cells, in agreement with previous findings ${ }^{31,32}$. This effect was significantly more dramatic in the absence of BRCA2 and was confirmed using two different MUS81 siRNAs (Fig. 5d and Supplementary Fig. 4a, b). Genetic knockdown-rescue experiments confirmed that complementation in MUS81-depleted U2OS cells with siRNA-resistant wild-type MUS81, but not with the catalytically inactive mutant, abrogated the effect of MUS81 depletion on replication fork stalling upon HU treatment (Fig. 5d).

MUS81 has two partners in human cells, EME1 and EME2, and the two proteins are thought to interact with MUS81 at different stages of the cell cycle. Genetic knockdown experiments showed that EME2, but not EME1, is involved in the same fork restart pathway, suggesting that the function of the MUS81EME2 complex is restricted to the S-phase (Supplementary Fig. $4 c, d)$, in agreement with previous findings ${ }^{32}$. MUS81 cleavage was previously shown to promote POLD3-dependent DNA synthesis at common fragile sites ${ }^{33}$ and telomeric ${ }^{34}$ loci in human cells. POLD3 is one of the accessory subunits of the replicative polymerase POL $\delta$ and was recently shown to be required for BIR, a specialized HR pathway to repair DSBs at stalled forks ${ }^{35}, 36$. Similar to MUS81 depletion, POLD3 loss increased fork stalling in BRCA2-deficient cells (Fig. 6a and Supplementary Fig. 4e). Moreover, it led to a severe defect in fork progression, suggesting that POLD3-dependent DNA synthesis is required to restart the MUS81-cleaved resected forks (Fig. 6b). Interestingly, POLD3/BRCA2 double depletion caused a further increase in DSB accumulation compared to depletion of BRCA2 alone and these DSBs were again rescued by MUS81 depletion confirming that POLD3 acts downstream of MUS81 in the pathway (Fig. 6c). The notion that BRCA2-deficient cells rely on the MUS81-dependent pathway to resume DNA synthesis is supported by our observation that cell viability is reduced in MUS81/BRCA2 co-depleted cells relative to BRCA2-depleted cells following $\mathrm{HU}$ treatment (Fig. 5e). Conversely, PARP

Fig. 3 Resected forks are able to restart in BRCA2-deficient cells and lead to increased chromosomal aberrations. a Expression of RAD51 after siRNA knockdown in U2OS cells. b Size distribution of IdU tract length in BRCA2-deficient and -proficient U2OS cells in the presence and absence of HU. Cells were transfected with control siRNA (siNEG), RAD51 siRNA, or BRCA2 siRNA before IdU and CldU labeling. Out of 2 repeats; $n \geq 250$ tracts scored for each data set. Bars represent the median. Statistics: Mann-Whitney; ${ }^{\star \star \star \star} P<0.0001$. c Schematic of the single-molecule DNA fiber tract analysis for the fork restart experiments. Red-green contiguous tracts (restarting forks). Red only tracts (stalled/terminated forks). d Quantification of restarting forks in BRCA2-deficient and -proficient U2OS cells with or without RAD51 siRNA knockdown. Out of 2 repeats, the percentage is established on at least 250 tracts scored for each data set. Mean shown. Errors, S.E.M. Statistics: unpaired $t$ test; ${ }^{\star \star} P<0.01$. e Quantification of restarting forks in BRCA2-deficient and -proficient U2OS cells with or without EXO1 siRNA knockdown. Mirin ( $50 \mu \mathrm{M})$ was added concomitantly with HU treatment, as indicated. Out of 2 repeats, the percentage is established on at least 250 tracts scored for each data set. Mean shown. Errors, S.E.M. f Size distribution of CldU tract length in BRCA2deficient and -proficient U2OS cells in the presence of HU. Cells were transfected with control siRNA (siNEG), EXO1 siRNA, or BRCA2 siRNA before IdU and CldU labeling. Mirin $(50 \mu \mathrm{M})$ was added concomitantly with $\mathrm{HU}$ treatment, as indicated. Out of 2 repeats; $n \geq 250$ tracts scored for each data set. Bars represent the median. Statistics: Mann-Whitney; ${ }^{\star \star \star \star} P<0.0001 . \mathbf{g}$ Chromosomal aberrations in BRCA2-deficient and -proficient U2OS cells in the presence of $\mathrm{HU}$. Left, representative images of metaphase spreads in the presence of HU. Scale bar, $25 \mu \mathrm{m}$. Sketch above the images delineates experimental design. Right, numbers of chromosomal aberrations per metaphase are plotted. At least 50 metaphases counted in each experiments. Mean shown, $n=3$.. Errors, S.E.M. Statistics: unpaired $t$ test: ${ }^{\star \star \star \star} P<0.0001$ 
a

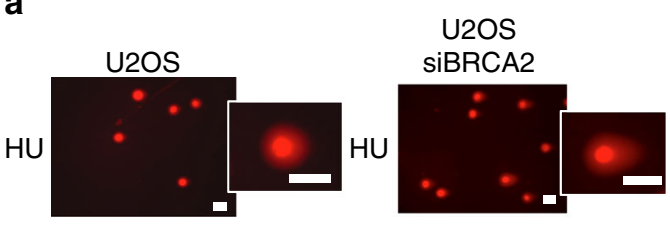

b

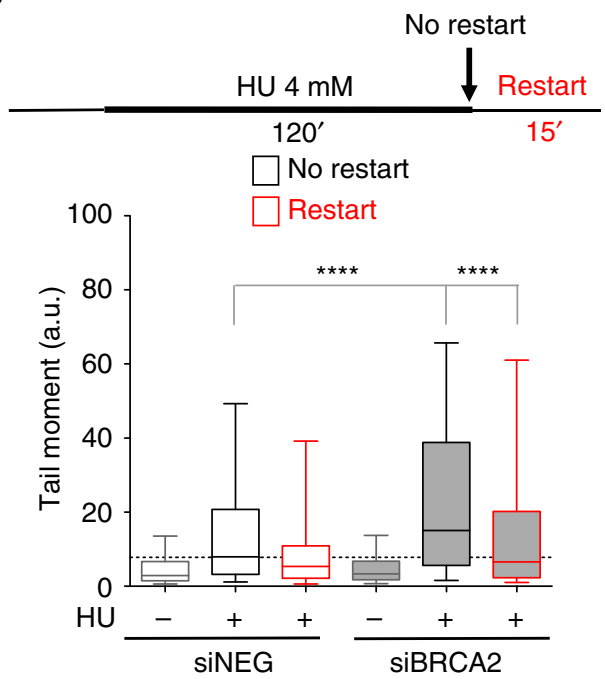

C

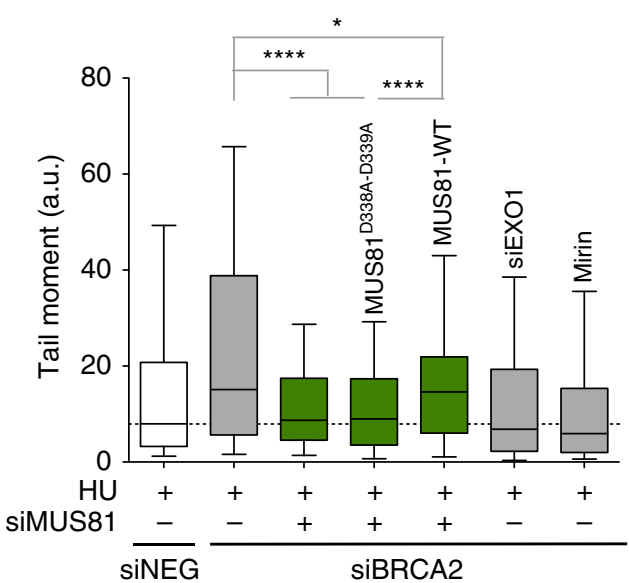

Fig. 4 MUS81 cleavage leads to transient DSB accumulation in BRCA2deficient cells. a Representative comet images of BRCA2-proficient and -deficient cells following treatment with $4 \mathrm{mM} \mathrm{HU}$ for $120 \mathrm{~min}$. Scale bar, $50 \mu \mathrm{m}$. b Neutral Comet assay monitoring DSB formation in BRCA2deficient and -proficient U2OS cells following HU treatment for $120 \mathrm{~min}$ (no restart) and 15 min after HU removal (restart). Cells were transfected with control siRNA (siNEG) or BRCA2 siRNA. Out of 3 repeats; $n \geq 200$ comets scored for each data set. Whiskers the 10th and 90th percentiles. ${ }^{\star \star \star \star} P<$ 0.0001 (Mann-Whitney test). c Neutral Comet assay monitoring DSB formation upon MUS81 depletion or complementation wild-type (MUS81WT) or catalytically dead (MUS81 D338A-D339A) MUS81. Cells were transfected with control siRNA (siNEG), BRCA2 siRNA, MUS81 siRNA, or EXO1 siRNA. MUS81-depleted cells were complemented with wild-type (MUS81-WT) or catalytically dead (MUS81 D338A-D339A) MUS81, when indicated. Mirin $(50 \mu \mathrm{M})$ was added concomitantly with $\mathrm{HU}$ treatment, as indicated. Out of 3 repeats; $n \geq 200$ comets scored for each data set Whiskers the 10th and 90th percentiles. ${ }^{\star \star \star \star} P<0.0001,{ }^{\star} P<0.05$ (Mann-Whitney test) inhibitor sensitivity was not significantly affected by MUS81 depletion consistent with the notion that PARP inhibitors, differently from $\mathrm{HU}$, prevent reversed fork accumulation ${ }^{10,13}$ and thereby do not require a MUS81-dependent pathway of fork rescue (Supplementary Fig. 4f). Collectively, these results suggest that BRCA2-deficent cells rely on a MUS81/POLD3-dependent mechanism to rescue resected replication forks following treatment with genotoxic agents that induce replication fork reversal and degradation in a BRCA2-deficient background.

MUS81 does not rescue forks in BRCA1-deficient cells. Our data suggest that BRCA1 shares a function similar to BRCA2 in reversed fork protection (Fig. 1). However, the MUS81 pathway is not required to rescue forks in BRCA1-deficient cells (Supplementary Fig. $4 \mathrm{~g}, \mathrm{~h}$ ), suggesting that different pathways mediate the restart of the resected forks depending on the particular genetic background. These findings are in agreement with recent studies, suggesting that MUS81 depletion differentially affects chemotherapeutic sensitivity in BRCA2- versus BRCA1-deficient cells (Alan D'Andrea personal communication) and with our immunofluorescence experiments showing that MUS81 and POLD3 foci accumulate specifically in BRCA2-, but not in BRCA1-deficient cells (Fig. 6d).

\section{Discussion}

This work defines the mechanism that leads to the extensive fork degradation phenotype observed in BRCA2-deficient cells and provides novel insights into the molecular steps that rescue the resected forks upon drug removal. We propose a model whereby BRCA2 protects the regressed arms of replication forks that have reversed upon drug treatment from nucleolytic degradation. In its absence, the double-stranded ends formed by fork reversal are targeted by the CtIP, MRE11, and EXO1 nucleases to initiate the degradation of the stalled replication intermediates (Fig. 6e). Recent data suggest that RAD51 promotes reversed fork formation $^{14}$ and is enriched on nascent DNA independently of BRCA $2^{5}$. We propose that RAD51 has two distinct functions during replication stress: a BRCA2-independent function in promoting the initial step of reversed fork formation and a BRCA2-dependent function, whereby BRCA proteins protect the already formed reversed forks from nucleolytic degradation by stabilizing the RAD51 filament on the regressed arm. In BRCA2deficient cells, this second function is lost, leading to the nascent strand degradation phenotype observed with BRCA2 mutants unable to stabilize RAD51 on ssDNA ${ }^{6}$ or with RAD51 mutants that destabilize the RAD51 nucleofilament ${ }^{37,38}$. Because of the severe defect in fork restart associated with RAD51 depletion are reported here, we also suggest that fork remodeling is a central mechanism of replication stress response following prolonged drug treatment. Collectively, these findings shed light on the longdebated function of central HR factors in fork remodeling and go beyond the oversimplified concept that HR factors are simply required for DSB repair during replication stress. Our findings also reveal that MUS81-dependent cleavage of the resected forks is required for fork restart in BRCA2-deficient cells. The discovery that replication stalling induces RAD51 foci formation in a MUS81-independent manner is consistent with the idea that MUS81 is required to cleave forks that have already undergone RAD51-dependent fork remodeling ${ }^{31}$. We propose that MUS81 acts downstream of MRE11- and EXO1-mediated degradation. On the basis of our findings that CtIP is required to promote fork resection and MRE11 endonuclease activity at the $5^{\prime}$ strand, we also propose that the initial degradation of the regressed arms generates a reversed fork with a $3^{\prime}$-ssDNA tail that is then cleaved by MUS81 to mediate fork restart. This notion is consistent with 


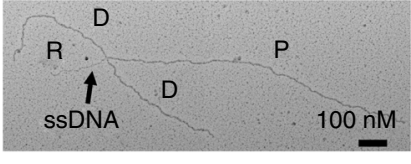

b

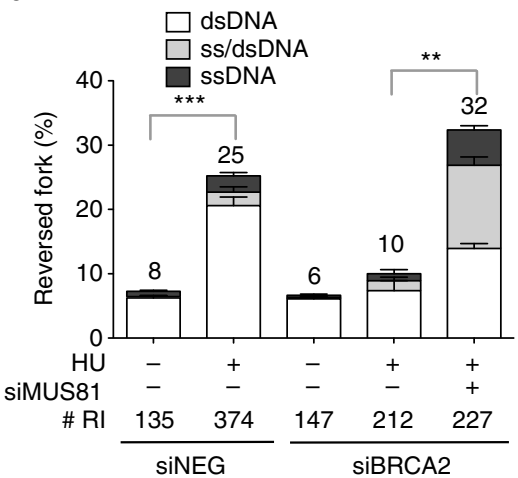

d

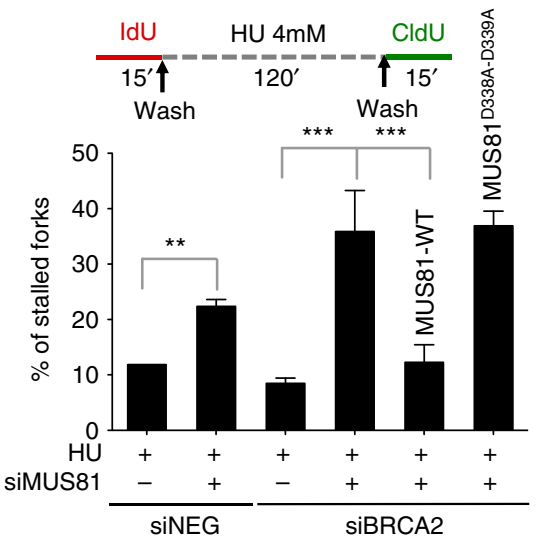

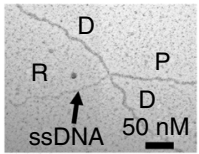

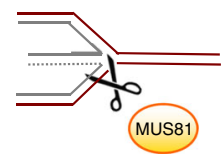

C

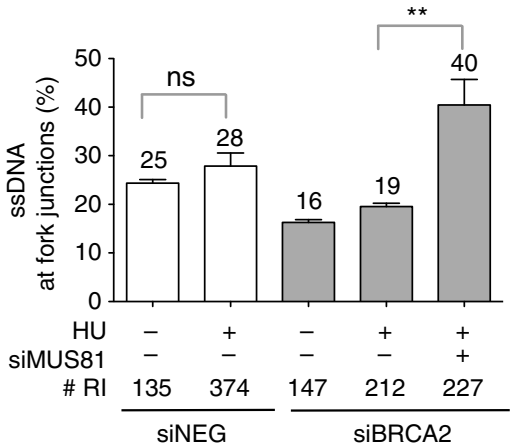

e

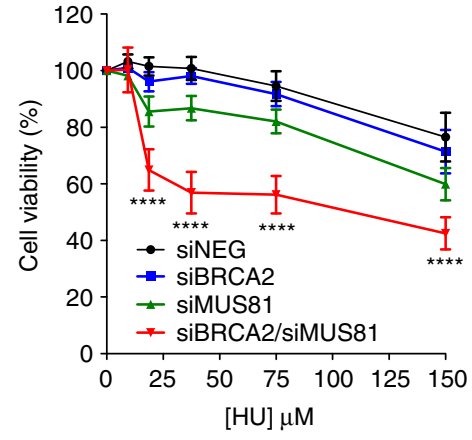

Fig. 5 MUS81 cleaves partially resected regressed forks with a ssDNA tail to promote fork rescue in BRCA2-deficient cells. D daughter strand, P parental strand, $R$ reversed arm. a Left, representative electron micrograph of a reversed fork with a single-stranded regressed arm. Center, magnified four-way junction at the reversed replication fork with a single-stranded regressed arm. The black arrow points to the ssDNA region on the regressed arm. Right, schematic model of the substrate cleaved by MUS81. b Frequency of fork reversal and sSDNA composition of the reversed arms in BRCA2-deficient U2OS cells treated with $4 \mathrm{mM} \mathrm{HU}$ for $5 \mathrm{~h}$. Cells were transfected with control siRNA (siNEG) or MUS81 siRNA. The percentage values are indicated on the top of the bar. "\# RI" indicates the number of analyzed replication intermediates. Mean shown, $n=3$. Errors, S.E.M. Statistics: unpaired $t$ test; ${ }^{\star \star} P<0.01$; ${ }^{\star \star \star} P<$ 0.001. c Percentage of forks with ssDNA at the fork junction in BRCA2-deficient U2OS cells treated with $4 \mathrm{mM}$ HU for $5 \mathrm{~h}$. Cells were transfected with control siRNA ( intermediates. Mean shown, $n=3$. Errors, S.E.M. Statistics: unpaired $t$ test; ${ }^{\star \star} P<0.01$. d Quantification of restarting forks in BRCA2-deficient and -proficient U2OS cells with or without MUS81 siRNA knockdown. MUS81-depleted cells were complemented with wild-type (MUS81-WT) or catalytically dead (MUS81D338A-D339A) MUS81, when indicated. Out of 3 repeats, the percentage is established on at least 250 tracts scored for each data set. Mean shown. Errors, S.E.M. Statistics: unpaired $t$ test; ${ }^{\star \star} P<0.01 ;{ }^{\star \star \star} P<0.001$ e Cell viability assays $72 \mathrm{~h}$ upon treatment with the indicated doses of HU. U2OS cells were transfected with control siRNA (siNEG), BRCA2 siRNA, or MUS81 siRNA. Mean shown, $n=6$. Errors, S.E.M. Statistics: two-way ANOVA, ${ }^{\star \star \star} P>$ $0.001 ;{ }^{\star \star \star \star} P>0.0001$ (differences between BRCA2 siRNA and BRCA2/MUS81 siRNA)

the in vitro data showing that MUS81 efficiently cleaves $3^{\prime}$ flaps, Y-shaped structures and nicked Holliday junctions ( $\mathrm{HJ})$, but has negligible activity toward intact $\mathrm{HJs}$ (resembling an intact reversed fork substrate $)^{30}, 39,40$. In the absence of MUS81 cleavage, the nucleolytic degradation might quickly proceed to degrade nascent strands behind the junction finally leading to the extensively resected forks observed by DNA fiber (Supplementary Fig. 4i). Recent studies suggest that completion of DNA replication at common fragile sites ${ }^{33}$ and telomeric ${ }^{34}$ loci occurs via a specialized form of DNA repair originally characterized in yeast and termed BIR, whereby MUS81 cleavage of stalled replication forks produces a migrating bubble that drives POLD3-dependent DNA synthesis $41,42,43$. We propose that a similar mechanism is responsible to rescue partially resected regressed forks in BRCA2deficient cells. We speculate that the MUS81/POLD3-dependent pathway used to rescue resected forks in BRCA2-deficient cells might represent a novel anticancer drug target specific for BRCA2-defective tumors to be used in combination with chemotherapeutics that cause replication fork reversal and degradation.

\section{Methods}

Cell lines and culture conditions. Cell lines: the human osteosarcoma U2OS cells (American Type Culture Collection), BRCA2-mutant ovarian cancer cells PEO1 (and the isogenic PEO4 cells, which contain a second point mutation restoring BRCA2 function) (provided by Dr. Lee Zou, Harvard Medical School) ${ }^{44}$, Fanconi 
a

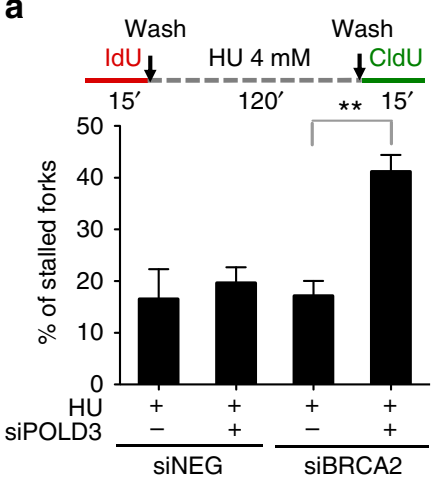

b
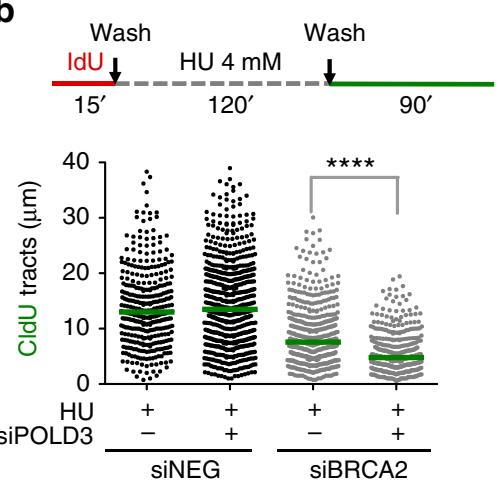

c

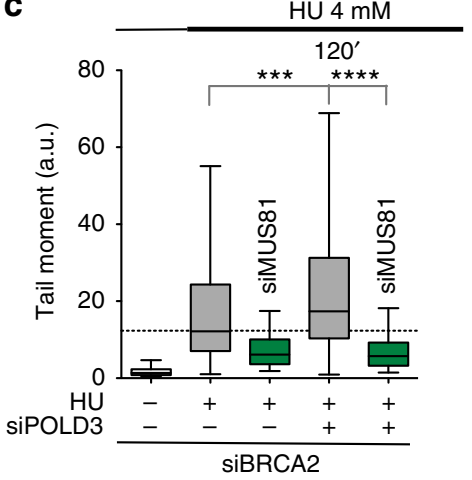

d
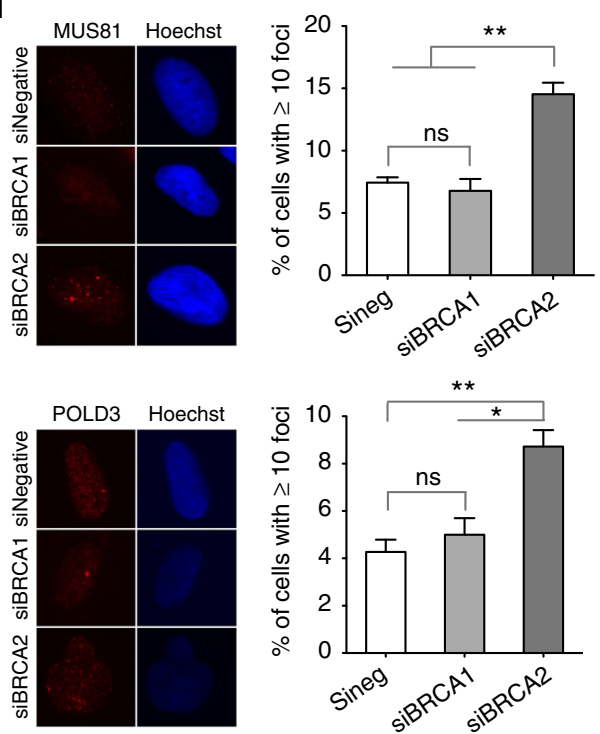

e
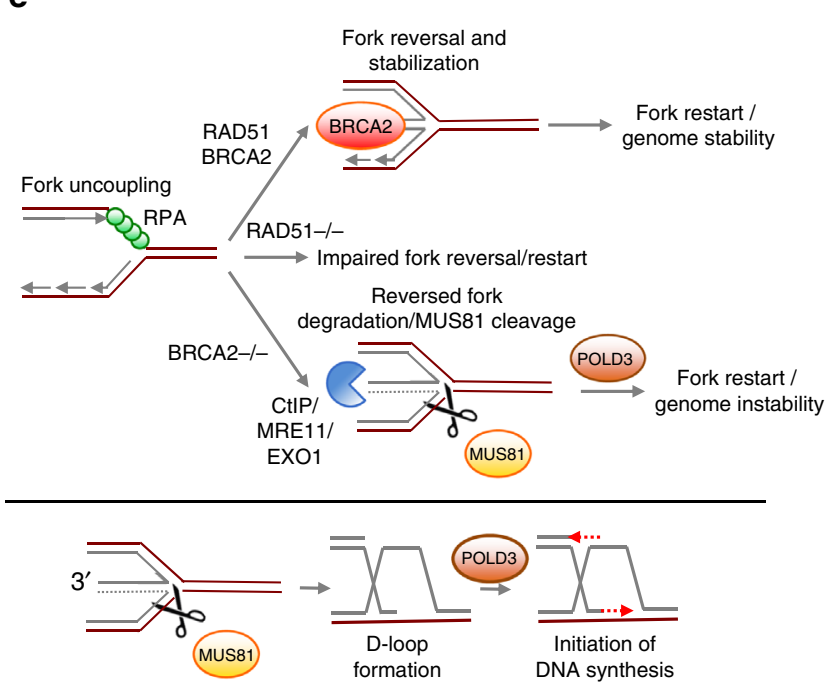

Fig. 6 POLD3 is required to restart resected forks in BRCA2-deficient cells. a Quantification of restarting forks in BRCA2-deficient and -proficient U2OS cells with or without POLD3 siRNA knockdown. Out of 2 repeats, the percentage is established on at least 250 tracts scored for each data set. Mean shown. Errors, S.E.M. Statistics: unpaired $t$ test; ${ }^{\star \star} P<0.01$. b Size distribution of CldU tract length in U2OS cells transfected with control siRNA (siNEG), POLD3 siRNA, or BRCA2 siRNA before IdU and CldU labeling. Out of 3 repeats; $n \geq 150$ tracts scored for each data set. Bars represent the median. Statistics: Mann-Whitney; ${ }^{\star \star \star \star} P<0.0001$. c Neutral Comet assay monitoring DSB formation upon POLD3 depletion in BRCA2-deficient cells. Cells were transfected with control siRNA (siNEG), BRCA2 siRNA, MUS81 siRNA, or POLD3 siRNA. Out of 2 repeats; $n \geq 200$ comets scored for each data set. Whiskers the 10 th and 90th percentiles. ${ }^{\star \star} P<0.001:{ }^{\star \star \star \star} P<0.0001$ (Mann-Whitney test). $\mathbf{d}$ Left, representative images of MUS81 and POLD3 foci observed upon HU treatment. Scale bar, $10 \mu \mathrm{m}$. U2OS cells were transfected with control siRNA (siNEG), BRCA1 siRNA, or BRCA2 siRNA and processed for immunofluorescence. Right, quantitation of MUS81 and POLD3 foci. Out of 3 repeats; $n \geq 150$ cells scored for each data set. Mean shown. Errors, S.E.M. Statistics: unpaired $t$ test; ${ }^{\star} P<0.05$; ${ }^{\star} P<<0.01$. e Top, roles of BRCA2, CtIP/MRE11/EXO1 and MUS81/POLD3 in reversed fork protection, degradation and cleavage/restart, respectively. Genotoxic agents lead to fork uncoupling and reversal. Reversed forks are stabilized by BRCA2, allowing accurate fork restart and genome stability. BRCA1 shares a similar function in reversed fork stabilization. RAD51 loss prevents fork reversal and compromises fork restart and cell viability. BRCA2 loss leads to CtIP/MRE11/EXO1-mediated degradation of the reversed replication forks. MUS81 cleaves the partially resected reversed forks with a sSDNA flap to grant POLD3-dependent fork restart and cell survival. Bottom, the MUS81 endonuclease cleaves the resected reversed forks with a ssDNA flap and lead to the subsequent formation of a D-loop structure that would in turn initiate POLD3-dependent DNA synthesis

anemia BRCA2-mutant line EUFA423 (and its derivative expressing wild-type BRCA2) (provided by Dr. Douglas Bishop, University of Chicago) ${ }^{45}, B R C A 1$ mutant ovarian cancer cell line UWB1.289 (and its complemented derivative expressing wild-type BRCA1) (provided by Dr. Lee Zou, Harvard Medical School) ${ }^{46}$. U2OS and EUFA $423 \mathrm{~F} / \mathrm{HAB} 2^{45}$ cells were grown in DMEM media supplemented with $10 \%$ fetal bovine serum (FBS), $100 \mathrm{U} \mathrm{ml}^{-1}$ penicillin and $100 \mu \mathrm{g} \mathrm{ml}^{-1}$ streptomycin at $37^{\circ} \mathrm{C}$ in $5 \% \mathrm{CO}_{2}$. Ovarian cancer PEO4/PEO1 ${ }^{44}$ were cultivated in RPMI media supplemented with $10 \%$ fetal bovine serum (FBS), $100 \mathrm{U} \mathrm{ml}^{-1}$ penicillin and $100 \mu \mathrm{g} \mathrm{ml}^{-1}$ streptomycin and UWB1/UWB1 + BRCA1 ${ }^{46}$ cells were grown in 50\% RPMI media, 50\% MEGM bullet kit (Lonza CC-3150) completed with $3 \% \mathrm{FBS}, 100 \mathrm{U} \mathrm{ml}^{-1}$ penicillin and $100 \mu \mathrm{g} \mathrm{ml}^{-1}$ streptomycin at $37^{\circ} \mathrm{C}$ in $5 \% \mathrm{CO}_{2}$.

Drug and reagents. The MRE11 inhibitor Mirin was from Sigma-Aldrich. The NSC-105808 DNA2 inhibitor was a gift from Gregorz $\operatorname{Ira}^{24}$. The DNA2 inhibitor was used at a concentration of $0.3 \mu \mathrm{M}$ for $24 \mathrm{~h}$. Hydroxyurea (Sigma-Aldrich) was dissolved in double-distilled $\mathrm{H}_{2} \mathrm{O}$ to obtain a $100 \mathrm{mM}\left(7.6 \mathrm{mg} \mathrm{ml}^{-1}\right)$ solution. Cisplatin (Sigma-Aldrich) was dissolved in PBS $10 \times$ to obtain a $5 \mathrm{mM}$ stock. UV-C was used at $40 \mathrm{~mJ} \mathrm{~cm}^{-2}$ as described in the labeling scheme. The MUS81 vectors used for the genetic complementation experiments was a gift from by Ian Hick$\operatorname{son}^{33}$. All vectors were amplified in DH5 $\alpha$ Escherichia coli and extracted with GeneJET Plasmid Midiprep Kit (ThermoFisher Scientific).

RNA interference. All transient gene depletions were carried out using the Lipofectamine RNAiMax transfection reagent (Life Technologies), except for EXO1 gene silencing that was performed using TransIT-siQuest (Mirus). SMARTpool siRNA from Dharmacon were employed to deplete the following genes: BRCA2 (L003462-00, $10 \mathrm{nM}, 24$ or $48 \mathrm{~h}$ ) as described ${ }^{47}$, BRCA1 (L-003461-00, $\left.50 \mathrm{nM}, 48 \mathrm{~h}\right)$ as described ${ }^{48}$, MRE11A (L-009271-00, $40 \mathrm{nM}, 48 \mathrm{~h}$ ) as described ${ }^{49}$, MUS81 (siRNA2: L-016143-01, $20 \mathrm{nM}, 48 \mathrm{~h}$ ) as described ${ }^{50}$, POLD3 (L-026692-01, $50 \mathrm{nM}$, 
$48 \mathrm{~h}$ ), as described ${ }^{33}$. The following genes were depleted with siRNA purchased from Ambion: DNA2 (4390827, $10 \mathrm{nM}, 48 \mathrm{~h}$ ), EXO1 (\#1: 4392420 and \#2: S17502, $40 \mathrm{nM}, 48 \mathrm{~h}$ ), RAD51 (4390827, $50 \mathrm{nM}, 48 \mathrm{~h}$ ) and MUS81 in rescue experiments (AM16708, $25 \mathrm{nM}, 48 \mathrm{~h}$ ). The EME2 siRNA was purchased from Qiagen (GeneSolution 146956, $80 \mathrm{nM}, 48 \mathrm{~h}$ ). The EME2 and CtIP siRNAs were custom made: EME2 (5'-GCGAGCCAGUGGCAAGAGA-3', $40 \mathrm{nM}, 48 \mathrm{~h}$ ) as described ${ }^{32}$ and CtIP (5'-GCUAAAACAGGAACGAAUC-3', $50 \mathrm{nM}, 48 \mathrm{~h}$ ) as described ${ }^{20}$. Silencer select negative control siRNA (4390843, Ambion) was used for the control experiments.

RT-qPCR and western blot analysis. The levels of siRNA-mediated knockdowns were determined by RT-qPCR or western blot. mRNAs was extracted with the PureLink RNA mini kit (Invitrogen) and CDNA was synthesized using the M-MLV reverse Transcriptase (Life Technologies) according to the manufacturer's indications. RT-qPCR experiments were performed using the following primers: BRCA1 (5'- AGAAACCACCAAGGTCCAAAG-3' and 5'-GGGCCCATAGCAACAGATTT-3'), BRCA2 (5'- AGGACTTGCCCCTTTCGTCTA-3' and $5^{\prime}$-TGCAGCAATTAACATATGAGG-3'), CtIP (5'-AAGAGGAGGAATTGTCTACTGC-3' and $5^{\prime}$-AGAATCTTGTCCCCTGTGGTGGA-3'), DNA2 (5'- ATTAGCATTTGGCGTGTGGC- $3^{\prime}$ and $5^{\prime}$-CTTTCTGTGTTACCCCCGGT- $3^{\prime}$ ), EME1 (5'-CTCATCCCTGAGGGCTAGAA- $3^{\prime}$ and $5^{\prime}$-AGTTGAAAGAGTGGCGGGA$3^{\prime}$ ), EME2 (5'-AGGTGGAAGAGGCCCTGGTA-3' and 5'-CCCTGCTGTGCAGAAGGAGA-3'), EXO1 (5' - CCTCGTGGCTCCCTATGAAG-3' and 5'-AGGAGATCCGAGTCCTCTGTAA-3'), GAPDH (5'- GAGCCACATCGCTCAGAC-3' and 5'-GACCAGGCGCCCAATAC-3'), MRE11 (5'- CCAGA-

GAAGCCTCTTGTACG-3' and 5'-TTCCACCTCTTCGACCTCTTC- $3^{\prime}$ ), MUS81 (5'- CTAACGAGAGGAGAGCCTGC- $3^{\prime}$ and $5^{\prime}$-GAGTGGAGCCAAGGGAAAAGA-3'), and POLD3 (5'- GAGTTCGTCACGGACCAAAAC-3' and 5'GCCAGACACCAAGTAGGTAAC-3'). Reactions were realized using the iQTM SYBR ${ }^{\circledR}$ Green supermix (Bio-Rad) following to the manufacturer's instructions. For each sample, normalization was performed using GAPDH. Results were expressed relative to indicated controls.

For western blot analysis, cells were lysed in a buffer containing $100 \mathrm{mM}$ Tris$\mathrm{HCl}, 4 \%$ SDS, $20 \%$ glycerol, $\beta$-mercaptoethanol $\left(100 \mu \mathrm{ml}^{-1}\right) .20 \mu \mathrm{g}$ of protein extracts were loaded onto a NuPAGE ${ }^{\mathrm{m}}$ Novex ${ }^{\mathrm{mm}} 3-8 \%$ Tris-Acetate Protein Gels, $1.0 \mathrm{~mm}, 15$-well (ThermoFisher Scientific). Proteins were transferred onto a nitrocellulose membrane (GE HealthCare) for $1 \mathrm{~h}$ at $15 \mathrm{~V}$ using the dry transfer machine Pierce G2 (ThermoFisher Scientific) following the manufacturer's instruction. Membranes were blocked for $1 \mathrm{~h}$ in TBS containing $0.1 \%$ Tween 20 . Next, membranes were probed with the anti-RAD51 rabbit polyclonal (1:1000; 05530-I Sigma-Aldrich), anti-EXO1 rabbit polyclonal (1:1000; provided by Dr. Zhongsheng You), anti-BRCA1 mouse monoclonal (1:1000; ab16781 Abcam), antiBRCA2 mouse monoclonal (1:1000; OP95 EMD-Millipore), anti- $\beta$-actin HRP (1:5000; A3854 Sigma-Aldrich) or anti- $\beta$ tubulin rabbit polyclonal (1:5000; sc-9104, Santa Cruz Biotechnology, Inc.) antibodies. Proteins were visualized using ECL (Pierce) according to the manufacturer's instructions.

DNA fiber assay. Briefly, asynchronously growing cells were labeled with two thymidine analogs: $20 \mu \mathrm{M}$ 5-iodo-2'-deoxyuridine (IdU; Sigma-Aldrich) followed by $200 \mu \mathrm{M}$ 5-chloro-2'-deoxyuridine (CldU; Sigma-Aldrich) for the indicated times ${ }^{13}, 51$. Cells were washed twice with PBS after the first pulse and treated with the indicated doses of the genotoxic agents. After the indicated times, cells were collected and resuspended in PBS at 100,000 cells per ml. A total of $2 \mu \mathrm{l}$ of this cell solution was mixed with $8 \mu \mathrm{l}$ of lysis buffer $(200 \mathrm{mM}$ Tris. $\mathrm{HCl} \mathrm{pH} \mathrm{7.5;50} \mathrm{mM}$ EDTA; $0.5 \%$ SDS) on a glass slide. After 6 min, the slides were tilted at a $20-45^{\circ}$ angle, and the resulting DNA spreads were air dried, fixed in 3:1 methanol/acetic acid and stored at $4{ }^{\circ} \mathrm{C}$. The DNA fibers were denatured with $2.5 \mathrm{M} \mathrm{HCl}$ for $1 \mathrm{~h}$, washed with PBS, and blocked with 5\% BSA in PBS/ 0.1\% Tween 20 for $1 \mathrm{~h}$. DNA immunostaining was performed with rat anti-BrdU antibody (1:50; AbCys SA, ABC117 7513) for CldU and mouse anti-BrdU antibody (1:50; Becton Dickson, 347580) for IdU in a humid chamber at $37^{\circ} \mathrm{C}$ for $1 \mathrm{~h}$. The following secondary antibodies were used: anti-rat Alexa 488 (1:100; Molecular Probes, A21470) and anti-mouse Alexa 546 (1:100; Molecular Probes, A21123) at $37^{\circ} \mathrm{C}$ for $45 \mathrm{~min}$. The slides were air dried and mounted with Prolong Gold Antifade reagent (Invitrogen). Images were sequentially acquired (for double-label) with LAS AF software using TCS SP 5 confocal microscope (Leica). A $63 \times / 1.4$ oil immersion objective was used. Images were captured at room temperature. The DNA tract lengths were measured using ImageJ and the pixel length values were converted into micrometers using the scale bars created by the microscope. $n \geq 150$ fiber tracts scored for each data set. The statistics for all these experiments measuring changes in the size of the IdU or CldU tracts were calculated on the total number of DNA tracts measured in each given sample (usually $n \geq 250$ ). For the fork restart experiments, the percentage of stalled forks was calculated on the basis of at least 250 tracts counted in each independent experiment. All DNA fiber experiments were performed in duplicate or triplicate, as indicated in the figure legends. Additional information on the minimal number of tracts that should be measured for a reliable estimation of changes in fork speed within a given sample can be found in refs. 26,52 .
Electron microscopy. For the EM analysis of replication intermediates, 5-10 $\times 10^{6}$ U2OS or PEO1/4 cells were collected and genomic DNA was cross-linked by two rounds of incubation in $10 \mu \mathrm{g} \mathrm{ml}^{-1} 4,5^{\prime}, 8$-trimethylpsoralen (Sigma-Aldrich) and $3 \mathrm{~min}$ of irradiation with $366 \mathrm{~nm}$ UV light on a precooled metal block ${ }^{10,26}$. Cells were lysed and genomic DNA was isolated from the nuclei by proteinase $\mathrm{K}$ (Roche) digestion and phenol-chloroform extraction. DNA was purified by isopropanol precipitation, digested with PvuII HF in the proper buffer for $3-5 \mathrm{~h}$ at $37^{\circ} \mathrm{C}$ and replication intermediates were enriched on a benzoylated naphthoylated DEAE-cellulose (Sigma-Aldrich) column. EM samples were prepared by spreading the DNA on carbon-coated grids in the presence of benzyl-dimethylalkylammonium chloride and visualized by platinum rotary shadowing. Images were acquired on a transmission electron microscope (JEOL 1200 EX) with sidemounted camera (AMTXR41 supported by AMT software v601) and analyzed with ImageJ (National Institutes of Health). EM analysis allows distinguishing duplex DNA - which is expected to appear as a $10 \mathrm{~nm}$ thick fiber, after the platimun/ carbon coating step necessary for EM visualization-from ssDNA, which has a reduced thickness of 5-7 nm. The criteria used for the unequivocal assignment of reversed forks include the presence of a rhomboid structure at the junction itself in order to provide a clear indication that the junction is opened up and that the fourway junction structure is not simply the result of the occasional crossing of two DNA molecules ${ }^{25}$. In addition, the length of the two arms corresponding to the newly replicated duplex should be equal $(b=c)$, whereas the length of the parental arm and the regressed arm can vary $(a \neq b=c \neq d)$. Conversely, canonical Holliday junction structures will be characterized by arms of equal length two by two $(a=b$, $\mathrm{c}=\mathrm{d})$.

Metaphase spreads. Cells were treated with $4 \mathrm{mM} \mathrm{HU}$ for $6 \mathrm{~h}$, washed twice with PBS, and released for $24 \mathrm{~h}$ in fresh medium. During the last $4 \mathrm{~h}, 10 \mu \mathrm{M}$ nocodazole was added to the medium. Cells were collected, washed and resuspended in $10 \mathrm{ml}$ of warmed hypotonic solution ( $10 \mathrm{mM} \mathrm{KCl}, 10 \% \mathrm{FBS})$ for $10 \mathrm{~min}$ at $37^{\circ} \mathrm{C}$. Cells were fixed by adding $500 \mu \mathrm{l}$ of cold fixation buffer (acetic acid 1: 3 ethanol). Cell pellets were washed four times with the cold fixation buffer and stored in this buffer at $4^{\circ} \mathrm{C}$ overnight. The nuclei were spread on cold slides. The slides were air dried overnight and mounted with Prolong Gold Antifade (Invitrogen) with DAPI. Images were acquired with a fluorescence microscope (LEICA DMU 4000B; 63×/ 1.40-0.60 NA oil) coupled to the LEICA DFC345FX camera. The images were analyzed with ImageJ. At least 50 metaphases per sample were scored in each experiment.

Neutral comet assay for DSB detection. A total of 700 cells were resuspended in $70 \mu \mathrm{l} 0.5 \%$ low melting point agarose (Trevigen, 4250-050-02) and spread on a comet slide (Trevigen, 4250-200-03). Cells were lysed in a cold lysis solution (Trevigen, $4250-050-01$ ) at $4{ }^{\circ} \mathrm{C}$ for $30 \mathrm{~min}$. DNA migration was performed in TBE buffer at $1 \mathrm{~V} \mathrm{~cm}^{-1}$ for $30 \mathrm{~min}$. Slides were washed in milliQ water, fixed with ethanol $70 \%$ for $30 \mathrm{~min}$ and dried at room temperature. Comets were labeled with SYBR $^{\circledast}$ Gold Nucleic Acid Gel Stain (ThermoFisher) for $30 \mathrm{~min}$. Images were acquired with a fluorescence microscope (LEICA DMU 4000B; 20×/0.4 CORR) coupled to the LEICA DFC345FX camera. The images were analyzed using ImageJ. At least 150 comets were scored per sample in each experiment.

Cell viability. Cell viability was determined using the Cell Proliferation Kit II (XTT, Roche) ${ }^{53}$. Briefly, cells were seeded at 13,000 cells per well in a 24 -well plate the day prior to treatment. Cells were treated chronically with the indicated doses of $\mathrm{HU}$ and cell viability was assessed 3 days after. The absorbance was measured at $450 \mathrm{~nm}$ with a reference wavelength at $650 \mathrm{~nm}$. Results were expressed as percentage of the untreated control.

Immunofluorescence microscopy. After treatment with $4 \mathrm{mM} \mathrm{HU}$, U2OS cells were extracted with $1 \times$ PBS containing $0.2 \%$ Triton X-100 and protease inhibitors (Pierce) for $5 \mathrm{~min}$ on ice prior to fixation with 3.2\% paraformaldehyde. The cells were then washed extensively with IF wash buffer $(1 \times$ PBS, $0.5 \%$ NP- 40 , and $0.02 \%$ $\mathrm{NaN}_{3}$ ), then blocked with IF Blocking Buffer (IF wash buffer plus 10\% FBS) for at least $30 \mathrm{~min}$. Anti-MUS81 mouse monoclonal (1:200; ab14387 Abcam) or antiPOLD3 mouse monoclonal (1:350; H00010714-M01 Abnova) antibodies were diluted in IF Blocking Buffer overnight at $4^{\circ} \mathrm{C}$. After staining with secondary antibodies (1:1000, conjugated with Alexa Fluor 594; A11032 Life Technologies) and Hoechst 33342 (1:1000, Sigma-Aldrich), samples were mounted using Prolong Gold mounting medium (Invitrogen). Epifluorescent microscopy was performed on an Olympus fluorescent microscope (BX-53) using a UPlanS-Apo 100×/1.4 oil immersion lens, Hamamatsu ORCA-Flash 4.0LT digital camera, and cell-Sens Dimension software. The raw images were exported into Adobe Photoshop, and for any adjustments in image contrast or brightness, the levels function was applied as previously described ${ }^{54}$. For foci quantitation, at least 150 cells were analyzed in triplicate.

Statistical analysis. Statistical analysis was performed using Prism (GraphPad Software). The statistical significance in each case was calculated as indicated in each figure legend. 
Data availability. The authors declare that all relevant data supporting the findings of this study are available with the article and its Supplementary Information files, or from the corresponding author upon request.

Received: 11 August 2017 Accepted: 23 August 2017

Published online: 16 October 2017

\section{References}

1. Antoniou, A. et al. Average risks of breast and ovarian cancer associated with BRCA1 or BRCA2 mutations detected in case Series unselected for family history: a combined analysis of 22 studies. Am. J. Hum. Genet. 72, 1117-1130 (2003).

2. King, M. C., Marks, J. H. \& Mandell, J. B., New York Breast Cancer Study, G. Breast and ovarian cancer risks due to inherited mutations in BRCA1 and BRCA2. Science 302, 643-646 (2003).

3. Metcalfe, K. et al. Family history of cancer and cancer risks in women with BRCA1 or BRCA2 mutations. J. Natl Cancer. Inst. 102, 1874-1878 (2010).

4. van Asperen, C. J. et al. Cancer risks in BRCA2 families: estimates for sites other than breast and ovary. J. Med. Genet. 42, 711-719 (2005).

5. Ray Chaudhuri, A. et al. Replication fork stability confers chemoresistance in BRCA-deficient cells. Nature 535, 382-387 (2016).

6. Schlacher, K. et al. Double-strand break repair-independent role for BRCA2 in blocking stalled replication fork degradation by MRE11. Cell 145, 529-542 (2011).

7. Schlacher, K., Wu, H. \& Jasin, M. A distinct replication fork protection pathway connects Fanconi anemia tumor suppressors to RAD51-BRCA1/2. Cancer Cell 22, 106-116 (2012).

8. Ying, S., Hamdy, F. C. \& Helleday, T. Mre11-dependent degradation of stalled DNA replication forks is prevented by BRCA2 and PARP1. Cancer Res. 72, 2814-2821 (2012)

9. Cannavo, E. \& Cejka, P. Sae2 promotes dsDNA endonuclease activity within Mre11-Rad50-Xrs2 to resect DNA breaks. Nature 514, 122-125 (2014).

10. Berti, M. et al. Human RECQ1 promotes restart of replication forks reversed by DNA topoisomerase I inhibition. Nat. Struct. Mol. Biol. 20, 347-354 (2013).

11. Berti, M. \& Vindigni, A. Replication stress: getting back on track. Nat. Struct. Mol. Biol. 23, 103-109 (2016).

12. Fugger, K. et al. FBH1 Catalyzes Regression of Stalled Replication Forks. Cell Rep. S2211-1247, 00174-00176 (2015).

13. Ray Chaudhuri, A. et al. Topoisomerase I poisoning results in PARP-mediated replication fork reversal. Nat. Struct. Mol. Biol. 19, 417-423 (2012).

14. Zellweger, R. et al. Rad51-mediated replication fork reversal is a global response to genotoxic treatments in human cells. J. Cell. Biol. 208, 563-579 (2015).

15. Bugreev, D. V., Rossi, M. J. \& Mazin, A. V. Cooperation of RAD51 and RAD54 in regression of a model replication fork. Nucleic Acids Res. 39, 2153-2164 (2011).

16. Hashimoto, Y., Ray Chaudhuri, A., Lopes, M. \& Costanzo, V. Rad51 protects nascent DNA from Mre11-dependent degradation and promotes continuous DNA synthesis. Nat. Struct. Mol. Biol. 17, 1305-1311 (2010).

17. Gravel, S., Chapman, J. R., Magill, C. \& Jackson, S. P. DNA helicases Sgs1 and BLM promote DNA double-strand break resection. Genes Dev. 22, 2767-2772 (2008).

18. Mimitou, E. P. \& Symington, L. S. Sae2, Exo1 and Sgs1 collaborate in DNA double-strand break processing. Nature 455, 770-774 (2008).

19. Nicolette, M. L. et al. Mre11-Rad50-Xrs2 and Sae2 promote $5^{\prime}$ strand resection of DNA double-strand breaks. Nat. Struct. Mol. Biol. 17, 1478-1485 (2010).

20. Sartori, A. A. et al. Human CtIP promotes DNA end resection. Nature 450, 509-514 (2007)

21. Zhu, Z., Chung, W. H., Shim, E. Y., Lee, S. E. \& Ira, G. Sgs1 helicase and two nucleases Dna2 and Exo1 resect DNA double-strand break ends. Cell 134, 981-994 (2008)

22. Vindigni, A. \& Lopes, M. Combining electron microscopy with single molecule DNA fiber approaches to study DNA replication dynamics. Biophys. Chem. 225, 3-9 (2017).

23. Spies, J. et al. Nek1 Regulates Rad54 to Orchestrate Homologous Recombination and Replication Fork Stability. Mol. Cell. 62, 903-917 (2016).

24. Ira, G. et al. Inhibition of DNA2 nuclease as a therapeutic strategy targeting replication stress in cancer cells. Oncogenesis 6, e319 (2017).

25. Neelsen, K. J., Chaudhuri, A. R., Follonier, C., Herrador, R. \& Lopes, M. Visualization and interpretation of eukaryotic DNA replication intermediates in vivo by electron microscopy. Methods Mol. Biol. 1094, 177-208 (2014).

26. Thangavel, S. et al. DNA2 drives processing and restart of reversed replication forks in human cells. J. Cell. Biol. 208, 545-562 (2015).

27. Anand, R., Ranjha, L., Cannavo, E. \& Cejka, P. Phosphorylated CtIP Functions as a Co-factor of the MRE11-RAD50-NBS1 Endonuclease in DNA End Resection. Mol. Cell. 64, 940-950 (2016).
28. Cannavo, E., Cejka, P. \& Kowalczykowski, S. C. Relationship of DNA degradation by Saccharomyces cerevisiae exonuclease 1 and its stimulation by RPA and Mre11-Rad50-Xrs2 to DNA end resection. Proc. Natl Acad. Scie. USA 110, E1661-E1668 (2013).

29. Nimonkar, A. V. et al. BLM-DNA2-RPA-MRN and EXO1-BLM-RPA-MRN constitute two DNA end resection machineries for human DNA break repair. Genes Dev. 25, 350-362 (2011).

30. Wyatt, H. D., Sarbajna, S., Matos, J. \& West, S. C. Coordinated actions of SLX1SLX4 and MUS81-EME1 for Holliday junction resolution in human cells. Mol. Cell 52, 234-247 (2013).

31. Hanada, K. et al. The structure-specific endonuclease Mus81 contributes to replication restart by generating double-strand DNA breaks. Nat. Struct. Mol. Biol. 14, 1096-1104 (2007).

32. Pepe, A. \& West, S. C. MUS81-EME2 promotes replication fork restart. Cell Rep. 7, 1048-1055 (2014).

33. Minocherhomji, S. et al. Replication stress activates DNA repair synthesis in mitosis. Nature 528, 286-290 (2015).

34. Dilley, R. L. et al. Break-induced telomere synthesis underlies alternative telomere maintenance. Nature 539, 54-58 (2016).

35. Costantino, L. et al. Break-induced replication repair of damaged forks induces genomic duplications in human cells. Science 343, 88-91 (2014).

36. Lydeard, J. R., Jain, S., Yamaguchi, M. \& Haber, J. E. Break-induced replication and telomerase-independent telomere maintenance require Pol32. Nature 448, 820-823 (2007).

37. Wang, A. T. et al. A Dominant Mutation in Human RAD51 Reveals Its Function in DNA Interstrand Crosslink Repair Independent of Homologous Recombination. Mol. Cell 59, 478-490 (2015).

38. Mijic, S. et al. Replication fork reversal triggers fork degradation in BRCA2defective cells. Nat. Commun. 8, doi:10.1038/s41467-017-01164-5 (2017).

39. Boddy, M. N. et al. Mus81-Eme1 are essential components of a Holliday junction resolvase. Cell 107, 537-548 (2001).

40. Whitby, M. C., Osman, F. \& Dixon, J. Cleavage of model replication forks by fission yeast Mus81-Emel and budding yeast Mus81-Mms4. J. Biol. Chem. 278, 6928-6935 (2003).

41. Donnianni, R. A. \& Symington, L. S. Break-induced replication occurs by conservative DNA synthesis. Proc. Natl Acad Sci. USA 110, 13475-13480 (2013).

42. Saini, N. et al. Migrating bubble during break-induced replication drives conservative DNA synthesis. Nature 502, 389-392 (2013).

43. Wilson, M. A. et al. Pif1 helicase and Poldelta promote recombination-coupled DNA synthesis via bubble migration. Nature 502, 393-396 (2013).

44. Sakai, W. et al. Functional restoration of BRCA2 protein by secondary BRCA2 mutations in BRCA2-mutated ovarian carcinoma. Cancer Res. 69, 6381-6386 (2009).

45. Howlett, N. G. et al. Biallelic inactivation of BRCA2 in Fanconi anemia. Science 297, 606-609 (2002)

46. DelloRusso, C. et al. Functional characterization of a novel BRCA1-null ovarian cancer cell line in response to ionizing radiation. Mol. Cancer Research: MCR 5 35-45 (2007).

47. Chun, J., Buechelmaier, E. S. \& Powell, S. N. Rad51 paralog complexes BCDX2 and $\mathrm{CX} 3$ act at different stages in the BRCA1-BRCA2-dependent homologous recombination pathway. Mol. Cell. Biol. 33, 387-395 (2013).

48. Morris, J. R. et al. The SUMO modification pathway is involved in the BRCA1 response to genotoxic stress. Nature 462, 886-890 (2009).

49. Ewald, B., Sampath, D. \& Plunkett, W. ATM and the Mre11-Rad50-Nbs1 complex respond to nucleoside analogue-induced stalled replication forks and contribute to drug resistance. Cancer Res. 68, 7947-7955 (2008).

50. Petermann, E., Orta, M. L., Issaeva, N., Schultz, N. \& Helleday, T. Hydroxyurea-stalled replication forks become progressively inactivated and require two different RAD51-mediated pathways for restart and repair. Mol. Cell 37, 492-502 (2010).

51. Quinet, A., Carvajal-Maldonado, D., Lemacon, D. \& Vindigni, A. DNA Fiber Analysis: Mind the Gap! Methods Enzymol. 591, 55-82 (2017).

52. Techer, H. et al. Replication dynamics: biases and robustness of DNA fiber analysis. J. Mol. Biol. 425, 4845-4855 (2013)

53. Quinet, A. et al. Gap-filling and bypass at the replication fork are both active mechanisms for tolerance of low-dose ultraviolet-induced DNA damage in the human genome. DNA Repair 14, 27-38 (2014).

54. Mosammaparast, N. et al. The histone demethylase LSD1/KDM1A promotes the DNA damage response. J. Cell. Biol. 203, 457-470 (2013).

\section{Acknowledgements}

We thank Joel Eissenberg, Yuna Ayala, and Anna Malkova for their careful reading of the manuscript and insightful comments. We are grateful to Ian Hickson (University of Copenhagen, Denmark) for providing MUS81 constructs. We are grateful to Douglas Bishop (University of Chicago) for providing the BRCA2-mutant EUFA423 cell line. We thank the Research Microscopy Core Facility of Saint Louis University for technical support. The work in the A.V. laboratory is supported by NIH grant R01GM108648 and 
by DOD BRCP Breakthrough Award BC151728. The work in the N.M. laboratory is supported by NIH grant R01CA193318. J.R.B. is supported by the Cell and Molecular Biology Training Grant (5T32GM007067-40).

\section{Author contributions}

D.L.: Designed, performed, and analyzed the DNA fibers, EM, comet and metaphase spreads analyses, with extensive technical assistance of J.J. for all the EM studies. A.Q.: Designed and conducted the DNA fiber experiments with the POLD3-depleted cells and the cell survival assays with the MUS81-depleted cells. J.R.B.: Conducted the immunofluorescence experiments. S.L., S.Y., Z.Y., G.I., N.M., and L.Z.: Provided crucial reagents ahead of publication and assisted with experimental design and manuscript finalization. A.V.: Designed and supervised the project and wrote the manuscript.

\section{Additional information}

Supplementary Information accompanies this paper at doi:10.1038/s41467-017-01180-5.

Competing interests: The authors declare no competing financial interests.

Reprints and permission information is available online at http://npg.nature.com/ reprintsandpermissions/
Publisher's note: Springer Nature remains neutral with regard to jurisdictional claims in published maps and institutional affiliations.

(c) (i) Open Access This article is licensed under a Creative Commons Attribution 4.0 International License, which permits use, sharing, adaptation, distribution and reproduction in any medium or format, as long as you give appropriate credit to the original author(s) and the source, provide a link to the Creative Commons license, and indicate if changes were made. The images or other third party material in this article are included in the article's Creative Commons license, unless indicated otherwise in a credit line to the material. If material is not included in the article's Creative Commons license and your intended use is not permitted by statutory regulation or exceeds the permitted use, you will need to obtain permission directly from the copyright holder. To view a copy of this license, visit http://creativecommons.org/ licenses/by/4.0/.

(C) The Author(s) 2017 